\title{
Desempeño de losas de concreto sobre terreno reforzadas con malla electrosoldada o fibras de acero
}

\author{
Performance of Concrete Slabs-on-Ground Reinforced \\ with Welded-Wire Mesh or Steel Fibers
}

\author{
Carrillo Julián \\ Universidad Militar Nueva Granada, UMNG, Bogotá, Colombia \\ Departamento de Ingeniería Civil \\ Correo:wjcarrillo@gmail.com \\ Silva Diego \\ Universidad Militar Nueva Granada, UMNG, Bogotá, Colombia \\ Departamento de Ingeniería Civil \\ Correo:diego.silva@gmail.com \\ Sánchez Martha \\ Universidad Militar Nueva Granada, UMNG, Bogotá, Colombia \\ Departamento de Ingeniería Civil \\ Correo:martha.sanchez@unimilitar.edu.co
}

Información del artículo: recibido: enero de 2016, aceptado: junio de 2016

\section{Resumen}

Las nuevas tendencias de materiales de alto desempeño ha motivado el uso de fibras de acero para mejorar el comportamiento del concreto. En el artículo se presentan los resultados de un proyecto de investigación experimental que busca evaluar el contenido de fibras de acero necesario para obtener un desempeño a flexión equivalente al de la losa de concreto sobre terreno reforzada con malla electrosoldada y con la cuantía mínima por contracción y temperatura. El programa experimental incluyó ensayos a flexión de 10 losas cuadradas de $600 \mathrm{~mm}$ de lado, $100 \mathrm{~mm}$ de espesor y diferente tipo de refuerzo; por ejemplo, losas con malla electrosoldada de alambres formados en frío con diámetro de $5 \mathrm{~mm}$ y aberturas cuadradas de $150 \times 150 \mathrm{~mm}$, losas con diferentes contenidos de fibra de acero $\left(5,9\right.$ y $\left.18 \mathrm{~kg} / \mathrm{m}^{3}\right)$, y losas de concreto simple. Las curvas carga-deflexión se utilizaron para determinar la absorción de energía asociada a diferentes valores de deflexión en el centro del claro de la losa. Los resultados de un análisis de regresión lineal se utilizaron para proponer el contenido equivalente de fibras de acero para remplazar, para un valor determinado de deflexión, el refuerzo convencional de la losa de concreto sobre terreno.

\section{Descriptores:}

- losa sobre terreno

- fibras de acero

- absorción de energía

- vivienda

- malla electrosoldada 


\begin{abstract}
New trends on high performance materials have motivated the use of steel fibers for improving concrete performance. The paper shows the results of an experimental research program aimed at evaluating the fiber content required to obtain a flexural performance equivalent to that of the slab-on-ground reinforced with with weldedwire mesh and the minimum steel ratio for shrinkage and temperature. The experimental program included flexural tests of 10 600-mm square concrete slabs, thickness of $100 \mathrm{~mm}$, and different type of reinforcement; i.e, slabs with welded-wire mesh having cold-drawn wires of $5 \mathrm{~mm}$-diameter and spacing of $150 \mathrm{~mm}$; slabs with different contents of steel fibers $\left(5,9\right.$ and $\left.18 \mathrm{~kg} / \mathrm{m}^{3}\right)$ and slabs with plain concrete. Load-deflection curves were used for computing the energy absorption related to different values of deflection at mid-span of slab. Results of a linear regression analysis were used to propose the equivalent fiber content to replace, for a particular value of deflection, the conventional reinforcement of the concrete slab-on-ground.
\end{abstract}

\section{Introducción}

En todo el mundo se han desarrollado métodos innovadores para optimizar las propiedades del concreto tales como la tenacidad, ductilidad y resistencia a compresión, a tensión, a cortante, así como a los cambios bruscos de temperatura. En cuanto al tipo de refuerzo utilizado, el concreto reforzado con fibras de acero (CRFA) se ha convertido en un material fundamental en la construcción de pisos industriales, pavimentos, tableros de puentes, elementos para la estabilización de taludes, revestimientos de túneles, elementos estructurales prefabricados, bóvedas y refractarios, entre otros (Lugo, 2007). Cuando se usan fibras de acero no es necesario costear desperdicios ni tener en cuenta el corte, amarre y uso de espaciadores, ya que son procedimientos exclusivos del refuerzo convencional por medio de barras o malla electrosoldada. En cuanto al transporte, es mucho más sencillo y económico el caso de las fibras, pues no es necesario el uso de herramientas especiales para su manejo en obra.

En el caso de las losas sobre terreno para viviendas también es necesario encontrar un método de reforzamiento más eficiente que el refuerzo convencional por medio de barras o malla electrosoldada. En este artículo se presentan los resultados de un proyecto de investigación experimental que busca evaluar la dosificación de fibras de acero necesaria para obtener un desempeño equivalente al de la losa de concreto reforzada con la cuantía mínima por contracción y temperatura establecida en la sección 7.12.2 de los Reglamentos ACI 318-11 y NSR-10 para losas sobre terreno ( $0.18 \%)$. Los ensayos se realizaron en el laboratorio de materiales y estructuras de la Universidad Militar Nueva Granada en Colombia. En general, el estudio busca plantear una alternativa constructiva para losas sobre terreno en viviendas donde actualmente se utiliza refuerzo convencional por medio de mallas electrosoldadas y donde las cargas de servicio son iguales o menores que $500 \mathrm{~kg} / \mathrm{m}^{2}$. El programa experimental incluye el ensayo de 10 losas cuadradas de $600 \mathrm{~mm}$ de lado y $100 \mathrm{~mm}$ de espesor distribuidas de la siguiente manera: dos losas de concreto simple (CS), dos losas con malla electrosoldada de alambres corrugados con diámetro de $5 \mathrm{~mm}$ y aberturas cuadradas de $150 \times 150 \mathrm{~mm}$, y seis losas con diferentes dosificaciones de fibra de acero $\left(5,9\right.$ y $18 \mathrm{~kg} / \mathrm{m}^{3}$, dos losas para cada dosificación). El desempeño de las losas se determinó a partir de la comparación de las curvas carga-deflexión y las curvas tenacidad-deflexión de las losas. A partir de las tendencias de los resultados se propone el contenido de fibras de acero que se podría utilizar para remplazar, para una determinada deflexión en el centro del claro de la losa, la malla electrosoldada en losas sobre terreno con cargas menores a $500 \mathrm{~kg} / \mathrm{m}^{2}$. En el estudio no se evalúa el efecto del tipo de suelo sobre el comportamiento de la losa.

\section{Comportamiento observado en losas sobre terreno}

Las fibras de acero se pueden caracterizar por medio de varios parámetros; uno de los principales es la relación de esbeltez o de aspecto, que se define como el cociente entre la longitud y el diámetro de la fibra, $l_{f} / d_{f}$. En CRFA, la dosificación, $D_{f}$, representa la masa de fibras por unidad de volumen. La fracción de volumen de fibras de acero, $V_{f}$, se utiliza para expresar la dosificación de forma adimensional y se calcula como el cociente entre $D_{f}$ y la densidad del acero de las fibras, $\gamma_{s} ;$ es decir, $7.850 \mathrm{~kg} / \mathrm{m}^{3}$. El parámetro $V_{f}$ se expresa usual- 
mente en porcentaje. Una de las principales propiedades mecánicas del CRFA es la tenacidad, que es una medida de la capacidad de absorber la energía durante la deformación. La tenacidad se puede estimar a partir del área bajo la curva carga-deformación. La tenacidad incrementa con la adición de fibras de acero, lo cual es útil para evitar una falla repentina del elemento bajo carga estática y mejora la absorción de energía en la carga dinámica (ACI 544.4R-88, 2009). La variable que más influye en la tenacidad es la capacidad adherente de las fibras (Banthia, 1995).

Varios estudios experimentales investigaron el comportamiento de losas de CRFA. Por ejemplo, Pigeon y Cantin (1998) realizaron ensayos en losas para investigar las propiedades a flexión del CRFA sometido a bajas temperaturas. El experimento se desarrolló a una temperatura que varió entre $-10^{\circ} \mathrm{C}$ y $-30^{\circ} \mathrm{C}$, y se utilizaron relaciones agua/cemento de $0.45,0.35$ y 0.30 , y fibras de acero con dosificaciones de $40 \mathrm{~kg} / \mathrm{m}^{3}$ y $60 \mathrm{~kg} / \mathrm{m}^{3}$. En el estudio de Pigeon y Cantin (1998) se observó que cuando la temperatura disminuye, la tenacidad del CRFA incrementa bajo carga a flexión. Los resultados de los experimentos de Khalloo y Afshari (2005) demostraron que las losas con concreto simple fallaron repentinamente y se agrietaron sin alguna deflexión apreciable. Sin embargo, las losas con CRFA se agrietaron gradualmente y posteriormente las losas fallaron. En general, Khalloo y Afshari (2005) observaron que en las losas con CRFA, la fuerza última a flexión no se incrementa significativamente cuando las fibras se agregan al concreto, pero la capacidad de absorción de energía mejora significativamente. Hadi (2008) ensayó una serie de losas cuadradas de $820 \mathrm{~mm}$ de lado y $80 \mathrm{~mm}$ de espesor, con cargas en el centro y soportadas con cuatro apoyos (uno en cada vértice). El objetivo de estudio fue comparar las propiedades de la losas de concreto con los diferentes tipos de fibras utilizadas (sin fibras, fibras de acero y fibras de polipropileno). En el estudio de Hadi (2008) se demostró que, antes del agrietamiento, la adición de fibras de acero al concreto no influye lo suficiente en la resistencia a flexión y rotura máxima del espécimen, y no hay cambios significativos en las características de flexión de las losas. Sin embargo, la deflexión última aumenta, el colapso tarda más que en la losa de CS y, por tanto, la absorción de energía del espécimen es mejor.

En cuanto a la comparación del tipo de refuerzo de las losas, Sorelli et al. (2004) compararon el comportamiento de losas sobre terreno para pavimentos industriales con dos tipos de refuerzo: fibras de acero y malla electrosoldada. En el estudio se incluyeron losas de CS, losas de CRFA con $V_{f}=0.38 \%$ y $V_{f}=0.57 \%$, y losas refor- zadas con malla electrosoldada de $8 \mathrm{~mm}$ de diámetro y aberturas cuadradas de $200 \times 200 \mathrm{~mm}$. Las losas de CS presentaron una carga última de flexión significativamente menor en comparación con las losas de CRFA y con las losas reforzadas con malla electrosoldada. En el estudio se observó que al aumentar el contenido de fibra, la capacidad de carga no aumenta significativamente, pero se obtiene un comportamiento más estable (poca degradación). Las losas con refuerzo convencional exhibieron mayor carga de rotura. Según Sorelli et al. (2004), las fibras de acero con gancho evitan el colapso frágil de la losa sobre terreno y proporcionan una notable resistencia al elemento. En el estudio se observó que una vez que aparece la primera grieta, se inicia una redistribución de tensión y la carga puede aumentar hasta que ocurre un mecanismo de colapso debido al puente de las fibras entre las fisuras. Los patrones de agrietamiento difieren significativamente en las losas con los dos tipos de refuerzo. Las fibras de acero no afectan las trayectorias de falla en la matriz del concreto de la losa, en donde se desarrollan dos grietas principales de forma diagonal a lo largo del espécimen. Por el contrario, la malla electrosoldada ocasiona grietas más estrechas y difusas que se desarrollan a lo largo de forma radial. De acuerdo con Nguyen et al. (2011), las fibras de acero mejoran considerablemente la resistencia al punzonamiento de las losas planas (losas apoyadas directamente sobre columnas), por ejemplo, con dosificaciones de 30 a $60 \mathrm{~kg} / \mathrm{m}^{3}$, la resistencia al punzonamiento aumenta de 9 a $40 \%$; este aumento es directamente proporcional al volumen de fibra. Además, este tipo de refuerzo reduce la anchura media de la grieta en las losas $71 \%$ en el estado límite de servicio. Por otro lado, las fibras aumentan la rigidez de las losas y mejoran la ductilidad.

\section{Programa experimental}

El programa experimental se desarrolló para determinar la dosificación de fibras de acero que se tendría que adicionar para que el desempeño de la losa sea equivalente al de las losas sobre terreno reforzadas con la cuantía mínima por contracción y temperatura establecida en la sección 7.12.2 de los Reglamentos ACI 318-11 y NSR-10, $\rho_{m i n}$ que es igual a $0.18 \%$. Por tanto, el ensayo principal de la investigación fue el ensayo de flexión de losas a partir del cual se determinó la tenacidad o la capacidad de absorción de energía. El programa experimental incluye 10 especímenes cuadrados de $600 \mathrm{~mm}$ de lado y $100 \mathrm{~mm}$ de espesor. El espesor de $100 \mathrm{~mm}$ corresponde al valor característico de losas sobre terreno para vivienda. Las variables del programa experi- 
mental son el tipo de refuerzo y la dosificación de fibras de acero. En cuanto al tipo de refuerzo se incluyeron dos losas sin refuerzo o concreto simple (CS), dos losas reforzadas con malla electrosoldada (CR) y seis losas con fibras de acero. En cuanto a la dosificación de fibras de acero se incluyeron dos losas de concreto reforzado con fibras en las siguientes dosificaciones: $5 \mathrm{~kg} / \mathrm{m}^{3}$ (CF-5), $9 \mathrm{~kg} / \mathrm{m}^{3}$ (CF-9) y $18 \mathrm{~kg} / \mathrm{m}^{3}$ (CF-18).

\section{Tipo de concreto y tipos de refuerzo}

El concreto que se utilizó para la investigación fue de peso normal con resistencia nominal a compresión de $21 \mathrm{MPa}$ y tamaño máximo de agregado de $12.7 \mathrm{~mm}(1 / 2$ pulg). Este tipo de concreto es el más utilizado para esta aplicación en viviendas. La composición del concreto por $\mathrm{m}^{3}$ fue: $283 \mathrm{~kg}$ de cemento, $71 \mathrm{~kg}$ de ceniza, $850 \mathrm{~kg}$ de arena, $896 \mathrm{~kg}$ de grava, $219 \mathrm{~kg}$ de agua y $2 \mathrm{~kg}$ de aditivo. El concreto se premezcló y suministró por la empresa Argos. En cuanto a las fibras de acero, se empleó una fibra de referencia Dramix Malla en Bolsa RL$45 / 50-\mathrm{BN}$, la cual tiene longitud $\left(l_{f}\right)$ de $50 \mathrm{~mm}$, diámetro $\left(l_{f}\right)$ de $1.05 \mathrm{~mm}$, relación de aspecto $\left.\left(l_{f} / d_{f}\right)\right)$ de 48 , y resistencia a tensión de 1.115 MPa. En la sección 7.12.2 de los Reglamentos ACI 318-11 y NSR-10 se indica que la cuantía mínima por contracción y temperatura en losas sobre el terreno es de $0.18 \%$. Para proporcionar esta cuantía, tal como se hace de forma convencional, en el estudio se utilizó una malla electrosoldada de alambres corrugados verticales y horizontales con diámetro de $5 \mathrm{~mm}$ y aberturas cuadradas de $150 \times 150 \mathrm{~mm}$.

Geometría, refuerzo y elaboración de especímenes

En la figura 1 se muestra la geometría y la configuración del refuerzo de las losas reforzadas convencionalmente con malla electrosoldada. Para mejorar el anclaje de las mallas se generó un gancho estándar en los extremos de la malla con un doblez a 90 grados, conforme a las especificaciones dadas en sección 7.2.3 de los Reglamentos ACI 318-11 y NSR-10. El recubrimiento de los alambres fue de $20 \mathrm{~mm}$, que es mayor al tamaño máximo nominal (TMN) del agregado utilizado $(12.7 \mathrm{~mm}$, $1 / 2$ pulg), tal como se especifica en la sección 7.2 de los Reglamentos ACI 318-11 y NSR-10.

Las losas se elaboraron en moldes metálicos. En las losas reforzadas con malla electrosoldada se colocaron espaciadores de $20 \mathrm{~mm}$ para garantizar el recubrimiento. Para prevenir el alineamiento preferencial de las fibras de acero dentro del concreto, el método de vibración de la matriz de CRFA fue diferente al del concreto simple y al del concreto reforzado con la malla electrosoldada. Para las losas de concreto simple (CS) y con malla electrosoldada se agregó una sola capa de concreto y se aplicaron 100 apisonadas con una barra de acero recta y con extremos redondeados. Para las losas de CRFA se vertió una sola capa de concreto y se aplicaron 100 golpes externos con un mazo de cabeza de goma. El desencofrado de las probetas se realizó a las $24 \pm 8$ horas. El curado se realizó inmediatamente después de desmoldar los especímenes, y consistió en la aplicación, sobre cada superficie de los especímenes, de una membrana de curado comercial.

\section{Configuración de ensayo de losas}

El ensayo de absorción de energía se realizó de acuerdo con los lineamientos de la norma NTC-5721 (2009). Los ensayos se realizaron en un marco de carga con capacidad de $100 \mathrm{kN}$, que cuenta con una rótula para garantizar la carga de forma axial. El marco dispone de un marco metálico cuadrado rígido y nivelado, con medidas internas de $500 \mathrm{~mm}$ de lado, con un borde perimetral sobre que se apoya el espécimen. El elemento donde se aplica la carga es una platina cuadrada de acero de 100 $\mathrm{mm}$ de lado y $20 \mathrm{~mm}$ de espesor (figura 2a). La velocidad de aplicación de la carga en el punto central de la losa fue de $1.5 \mathrm{~mm} / \mathrm{min}$. Para medir las deflexiones se utilizaron cinco transductores de desplazamiento lineal; cuatro de ellos estuvieron montados en un dispositivo especial patentado (Carrillo et al., 2015) que se sujeta en los extremos de la parte superior de la probeta. Estos cuatro transduc-

$(\mathrm{mm})$
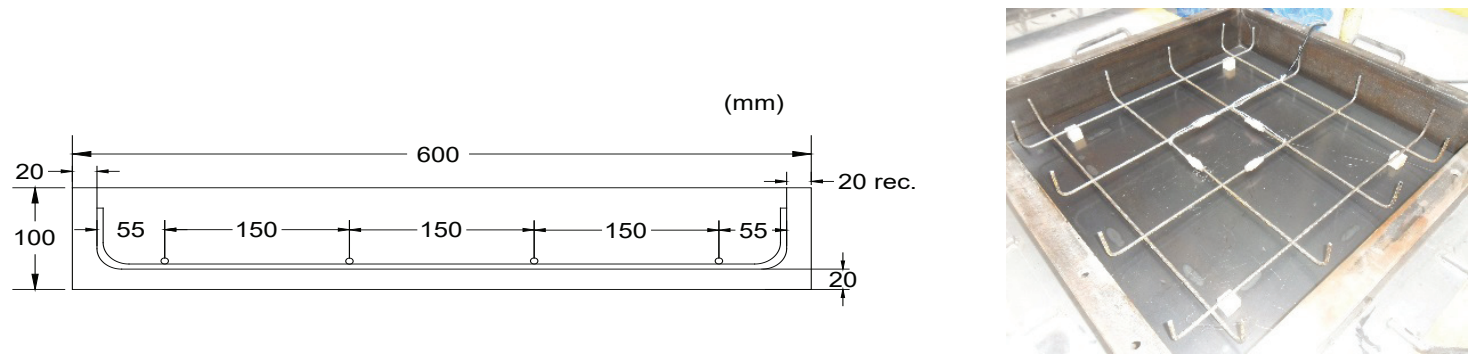

Figura 1. Geometría y configuración del refuerzo de la losa reforzada convencionalmente con malla electrosoldada: a) sección transversal, b) colocación de la malla 
tores de desplazamiento son de marca TML, referencia CDP-10, con capacidad máxima de $10 \mathrm{~mm}$. Adicionalmente se colocó un transductor de desplazamiento en el centro de la probeta. Este transductor es de marca Controls, referencia 82P0331/D1, con capacidad máxima de $10 \mathrm{~mm}$. Para medir la deformación de los alambres de las losas reforzadas con malla electrosoldada, previamente a la fundida se adhirieron a los alambres cuatro deformímetros marca TML, referencia FLA-2-11-5L, tal como se indica en la figura $2 b$. En los ensayos de flexión de losas se determinaron parámetros de desempeño tales como carga máxima $\left(P_{\max }\right)$, carga última asociada a la falla de la losa $\left(P_{u}\right)$, deflexión en $P_{u}\left(\delta_{u}\right)$ y tenacidad en flexión de losas $\left(T_{1}\right)$.

Para caracterizar las propiedades mecánicas del concreto se realizaron ensayos de compresión, módulo de elasticidad, tensión indirecta (compresión diametral) y flexión, con base en los lineamientos de las normas ASTM correspondientes. En general, el proyecto incluyó el ensayo de 36 especímenes de forma cilíndrica con $150 \mathrm{~mm}$ de diámetro y $300 \mathrm{~mm}$ de longitud, y 12 vigas de sección cuadrada con $150 \mathrm{~mm}$ de lado, y 600 $\mathrm{mm}$ de longitud. Para caracterizar las propiedades del concreto de cada losa, se realizaron una serie de ensayos de forma independiente. Los detalles de los ensayos para caracterizar las propiedades del concreto se presentan en Carrillo y Silva (2016).

La malla electrosoldada es un material compuesto de alambre de acero trabajado en frío, fabricado en paneles o rollos mediante el proceso de soldadura por resistencia eléctrica. En este estudio, las propiedades mecánicas de los alambres de la malla electrosoldada se caracterizaron a partir de ensayos de tensión, los cuales se realizaron en una Máquina Universal. Para determinar el límite de fluencia y la máxima resistencia a tensión de alambres de malla se siguieron los lineamientos de la norma NTC-3353 (2003).
Configuración de ensayos de alambres de acero

Para tener un índice de la capacidad de ductilidad de los alambres se calcularon parámetros tales como la reducción de área (norma norteamericana), doblado (norma colombiana) y alargamiento (norma mexicana). Para determinar la reducción de área se utilizó el procedimiento de la norma ASTM A-82 (2001), donde se indica que se debe unir manualmente la muestra fracturada y medir el diámetro en la sección transversal más pequeña. La diferencia entre el área original y la que se encontró después de la falla, expresada en porcentaje, representa la reducción del área. El ensayo de doblado se realizó según la norma NTC-1907 (2003), donde se indica que la probeta se debe doblar $90^{\circ}$ a temperatura ambiente, y no se debe presentar agrietamiento en la parte exterior. La probeta se dobla con un mandril con diámetro equivalente a dos veces el diámetro de la probeta. Finalmente, en la norma NMX B-253 (2006) se indica que el alargamiento se calcula como la diferencia entre la longitud original calibrada y la encontrada después de la fractura, expresada en porcentaje. Para calcular la curva completa esfuerzo-deformación se adhirieron a los alambres deformímetros marca TML, referencia FLA-2-11-5L; los mismos que se adhirieron en los alambres de las losas.

\section{Resultados y discusión}

En esta sección se presentan y discuten los esquemas de agrietamiento final de las losas, así como las curvas carga-deflexión de las losas para estimar la tenacidad con las diferentes dosificaciones de fibra de acero o con la malla electrosoldada. Adicionalmente, se presentan las propiedades del concreto en estado fresco, las propiedades mecánicas del concreto en estado endurecido
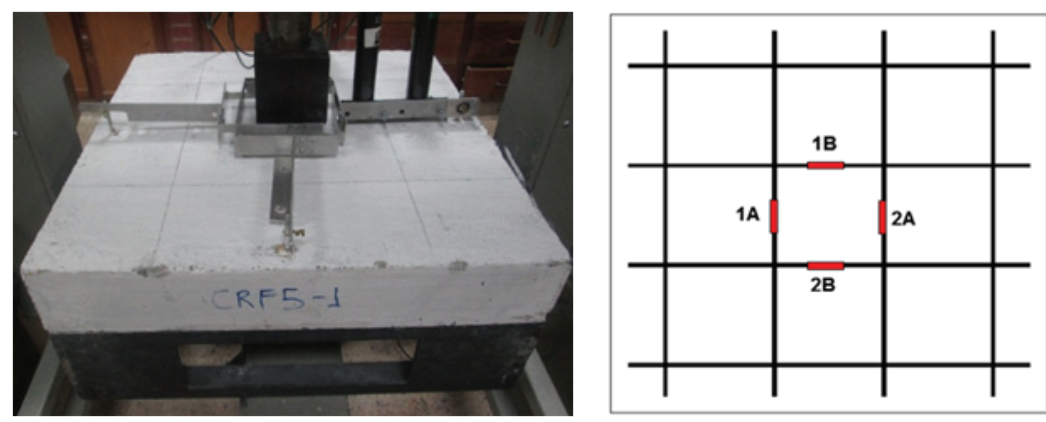

Figura 2. Configuración del ensayo a flexión de losas: a) dispositivo preparado, b) ubicación de deformímetros en los alambres 
y las propiedades mecánicas de la malla electrosoldada. Para estudiar los datos medidos se utilizaron parámetros estadísticos tales como el promedio $(X)$, desviación estándar $(S)$, coeficiente de variación $(C V)$ y coeficiente de correlación $(r)$. El coeficiente de variación $(C V)$ es directamente proporcional a la dispersión y representa la relación porcentual entre la desviación estándar $(S)$ y el promedio $(X)$. El coeficiente $r$ mide la intensidad de la relación lineal entre los valores calculados por una ecuación y los datos obtenidos experimentalmente.

\section{Propiedades de los materiales}

Durante los ensayos de compresión axial se obtuvieron los principales parámetros tales como resistencia a compresión, $f_{c^{\prime}}^{\prime}$ deformación unitaria asociada a $f_{c}^{\prime}, \varepsilon_{0 \prime}$ módulo de elasticidad, $E_{c}$, y relación de Poisson, $v$. En la figura 3a se muestran los principales parámetros asociados a la curva carga-deformación medida durante el ensayo de tensión por compresión diametral de cilindros, donde $f_{o}$ es el esfuerzo a tensión por compresión diametral, $f_{t}$ es el esfuerzo resistente máximo, $f_{p}$ es el esfuerzo resistente asociado al inicio de la contribución de las fibras, $f_{u t}$ es el esfuerzo resistente último a tensión por compresión diametral; $\varepsilon_{0^{\prime}} \varepsilon_{t}, \varepsilon_{p}$ y $\varepsilon_{u t}$ son las deformaciones unitarias asociadas a $f_{\alpha^{\prime}} f_{t}, f_{p}$ y $f_{u t}$, respectivamente, y $T_{t}$ es la tenacidad en compresión diametral. En la figura $3 \mathrm{~b}$ se muestran los principales parámetros asociados a la curva carga-deflexión medida durante el ensayo de tensión por flexión de vigas, donde $f_{r}$ es resistencia al agrietamiento o módulo de ruptura, $f_{l / 1300} \mathrm{y}$ $f_{l c / 150}$ son las resistencias residuales asociadas a deflexiones en el centro del claro equivalentes a $l_{c} / 300$ y a $l_{c} / 150$, respectivamente, $f_{u}$ es la resistencia última, $\delta_{r}$ es la deflexión asociada a $f_{r}, \delta_{u}$ es la deflexión última, y $T_{f}$ es la tenacidad en tensión por flexión en Joule (J). El punto último corresponde al punto donde colapsa el espécimen y ocurre una pérdida de resistencia súbita.

En la tabla 1 se muestra el resumen de las propiedades mecánicas del concreto. En dicha tabla se muestran los valores de dosificación real (valores medidos), que fueron clave para el análisis de la información experimental de los especímenes. Para propósitos del análisis de resultados, a partir de esta sección se utilizarán los valores reales (medidos) de la dosificación de fibras de acero. Los detalles de los resultados se presentan en Carrillo y Silva (2016).

En la tabla 2 se indica el valor mínimo de las propiedades mecánicas de los alambres de malla recomendado en diferentes normas. Para caracterizar las propiedades a tensión de los alambres de malla se utilizaron tres probetas (M1, M2 y M3) de $600 \mathrm{~mm}$ de longitud. Sin embargo, no se logró obtener un resultado de resistencia en la probeta M3 debido a un error del equipo en la adquisición de datos. En la figura 4a se muestran las curvas esfuerzo-deformación en tensión de los alambres de la malla electrosoldada.

Las propiedades y características medidas se indican en la tabla 3. La resistencia de fluencia $\left(f_{y}\right)$ y la deformación de fluencia $\left(\varepsilon_{y}\right)$ se determinaron con base en el método de corrimiento; es decir, trazando una línea paralela a la zona elástica de la curva en una deformación unitaria de $0.002(0.2 \%)$. En la tabla 3 se observa que la resistencia a la tensión $f_{u}$ y el alargamiento no cumplen estrictamente con los requerimientos establecidos en las normas descritas (diferencias con el valor mínimo cercanas a 5\%). Los demás parámetros sí cumplieron con los valores mínimos indicados en la normativa.

\section{Desempeño a flexión de losas}

En la figura 5a se muestran las curvas carga-deflexión medidas durante los ensayos a flexión de las losas de CS, CRFA y reforzadas con malla electrosoldada. La deflexión corresponde a la medida en el centro del claro de la losa. En la figura 6 se muestran los esquemas de agrietamiento final de las losas. Teniendo en cuenta que para cada tipo de losas se ensayaron dos especímenes, en la Figura 5a se muestra la curva del espécimen con el mejor desempeño. En la figura $4 \mathrm{~b}$ se muestran las curvas carga-deformación unitaria de los alambres de la malla medida por medio de los deformímetros adheri-
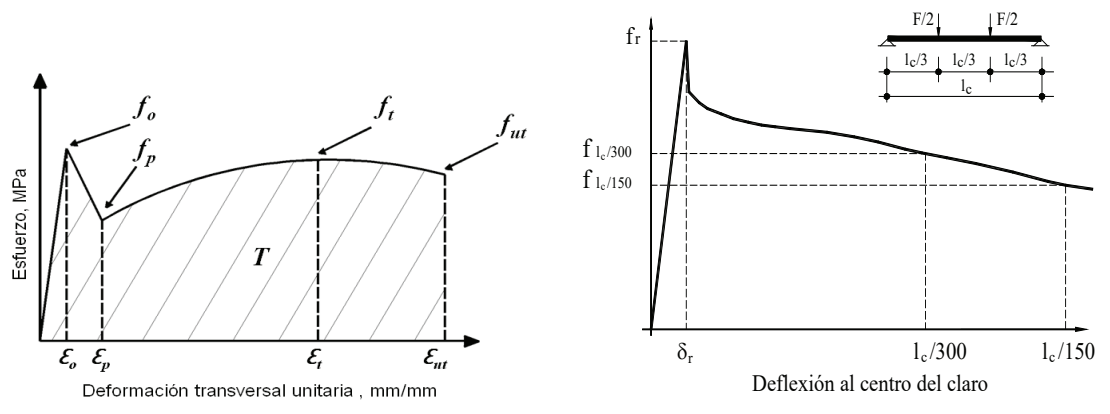

Figura 3. Curva característica: a) tensión indirecta, b) flexión en vigas 
dos en los alambres, tal como se indica en la figura $2 \mathrm{~b}$. En la figura $4 \mathrm{~b}$ se observa que, en el punto de resistencia máxima de la losa con refuerzo convencional, todos los alambres exhibieron deformaciones mayores a las de fluencia. En la tabla 4 se muestran los principales parámetros medidos en el ensayo a flexión de losas de CS, CRFA y concreto reforzado con malla electrosoldada (CR). En la tabla se incluyen los parámetros de carga y deflexión asociados a la carga máxima $\left(P_{\max } \delta_{\max }\right)$ y a la carga última $\left(P_{u^{\prime}} \delta_{u}\right)$. El punto último se definió para

Tabla 1. Propiedades mecánicas del concreto

\begin{tabular}{|c|c|c|c|c|c|c|}
\hline & $\begin{array}{c}D_{f}, \mathrm{~kg} / \mathrm{m}^{3} \\
V_{\mathrm{f}} \% \\
V_{f_{r}} \times\left(l_{f} / d_{f}\right)\end{array}$ & $\begin{array}{l}\text { Parámetro } \\
\text { estadístico }\end{array}$ & $\begin{array}{c}0 \\
0.00 \\
0.00 \\
\end{array}$ & $\begin{array}{c}3.4 \\
0.04 \\
1.92 \\
\end{array}$ & $\begin{array}{c}7.6 \\
0.10 \\
4.80 \\
\end{array}$ & $\begin{array}{l}14.1 \\
0.18 \\
8.64 \\
\end{array}$ \\
\hline \multirow{6}{*}{ 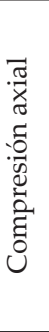 } & $f_{c^{\prime}} \mathrm{MPa}$ & $\begin{array}{c}X \\
\text { O }\end{array}$ & 28.7 & 30.2 & 31.2 & 31.7 \\
\hline & \multirow[b]{2}{*}{$\varepsilon_{0}\left(\right.$ asociada a $\left.f_{c}\right)$} & $X$ & 00018 & 00023 & 00025 & 0 0970 \\
\hline & & $\stackrel{\lambda}{C V}, \%$ & 5.5 & $\begin{array}{l}0.0023 \\
27.7\end{array}$ & $\begin{array}{l}0.0020 \\
15.8\end{array}$ & $\begin{array}{c}0.0028 \\
26.7\end{array}$ \\
\hline & $E_{c^{\prime}} \mathrm{MPa}$ & $\begin{array}{c}X \\
C V, \%\end{array}$ & $\begin{array}{c}17606 \\
3.9\end{array}$ & $\begin{array}{c}18034 \\
4.6\end{array}$ & $\begin{array}{c}17250 \\
2.3\end{array}$ & $\begin{array}{c}17600 \\
0.2\end{array}$ \\
\hline & \multirow{2}{*}{$v$} & $X$ & 0.16 & 0.18 & 0.16 & 0.16 \\
\hline & & $C V, \%$ & 3.5 & 6.2 & 3.5 & 8.6 \\
\hline \multirow{18}{*}{ 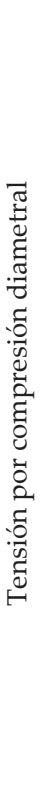 } & \multirow{2}{*}{$f_{o^{\prime}} \mathrm{MPa}$} & X & 2.91 & 2.97 & 3.03 & 3.03 \\
\hline & & $C V, \%$ & 3.8 & 4.5 & 1.7 & 4.2 \\
\hline & \multirow{2}{*}{$\varepsilon_{o}$} & X & 0.0002 & 0.0003 & 0.0003 & 0.0005 \\
\hline & & $C V, \%$ & 47.9 & 29.4 & 20.1 & 49.3 \\
\hline & \multirow{2}{*}{$f_{p^{\prime}}, \mathrm{MPa}$} & X & - & 2.8 & 2.8 & 3.1 \\
\hline & & $C V, \%$ & - & 0.4 & 1.7 & 7.2 \\
\hline & \multirow[b]{2}{*}{$\varepsilon_{p}$} & X & - & 0.0086 & 0.0045 & 0.0021 \\
\hline & & $C V, \%$ & - & 10.0 & 12.9 & 38.9 \\
\hline & \multirow{2}{*}{$f_{t^{\prime}} \mathrm{MPa}$} & X & - & 2.87 & 2.67 & 3.61 \\
\hline & & $C V, \%$ & - & 0.6 & 11.1 & 2.4 \\
\hline & \multirow[b]{2}{*}{$\varepsilon_{t}$} & X & - & 0.0145 & 0.0145 & 0.0165 \\
\hline & & $C V, \%$ & - & 4.9 & 29.4 & 18.9 \\
\hline & \multirow{2}{*}{$f_{u t}, \mathrm{MPa}$} & X & - & 2.05 & 2.20 & 2.86 \\
\hline & & $C V, \%$ & - & 2.7 & 6.4 & 2.4 \\
\hline & \multirow[b]{2}{*}{$\varepsilon_{u t}$} & X & - & 0.0304 & 0.0228 & 0.0207 \\
\hline & & $C V, \%$ & - & 7.6 & 7.4 & 9.7 \\
\hline & \multirow{2}{*}{$T_{t^{\prime}} \mathrm{J}$} & X & - & 283 & 346 & 355 \\
\hline & & $C V, \%$ & - & 19.5 & 36.9 & 3.7 \\
\hline \multirow{14}{*}{ 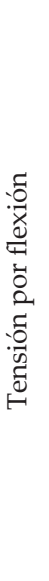 } & \multirow{2}{*}{$f_{r}=f_{\max }, \mathrm{MPa}$} & X & 4.2 & 4.2 & 4.2 & 4.3 \\
\hline & & $C V, \%$ & 1.4 & 0.3 & 3.7 & 7.9 \\
\hline & \multirow{2}{*}{$\delta_{r^{\prime}} \mathrm{mm}$} & X & 0.09 & 0.09 & 0.10 & 0.10 \\
\hline & & $C V, \%$ & 2.7 & 2.4 & 17.9 & 4.7 \\
\hline & \multirow{2}{*}{$f_{l c / 300} / f_{r}$} & X & - & 0.72 & 0.77 & 0.78 \\
\hline & & $C V, \%$ & - & 10.5 & 0.9 & 3.7 \\
\hline & \multirow{2}{*}{$f_{l c / 150} / f_{r}$} & X & - & 0.49 & 0.62 & 0.63 \\
\hline & & $C V, \%$ & - & 14.1 & 7.8 & 6.6 \\
\hline & \multirow{2}{*}{$f_{u}, \mathrm{MPa}$} & X & - & 0.44 & 0.46 & 0.85 \\
\hline & & $C V, \%$ & - & 46.9 & 32.1 & 58.9 \\
\hline & \multirow{2}{*}{$\delta_{u^{\prime}} \mathrm{mm}$} & X & - & 7.4 & 7.4 & 7.8 \\
\hline & & $C V, \%$ & - & 6.5 & 11.1 & 2.9 \\
\hline & \multirow{2}{*}{$T_{f,} \mathrm{~J}$} & $X$ & 1.6 & 112.1 & 125.9 & 131.0 \\
\hline & & $C V, \%$ & 11.0 & 2.7 & 17.9 & 2.6 \\
\hline
\end{tabular}


DOI: https://doi.org/10.1016/j.riit.2016.11.009

Tabla 2. Valores mínimos específicos

\begin{tabular}{lcc}
\hline \multicolumn{1}{c}{ Propiedades } & Valor mínimo & Norma \\
\hline Resistencia a fluencia, $f_{y^{\prime}} \mathrm{MPa}$ & 485 & NTC-3353 \\
Resistencia a la tensión, $f_{u^{\prime}} \mathrm{MPa}$ & 550 & NTC-3353 \\
Reducción de área, $\%$ & 30 & ASTM A-82 \\
Alargamiento, $\%$ & 6 & NMX B-253 \\
Doblado $90^{\circ}, \mathrm{Si} / \mathrm{No}$ & - & NTC-1907 \\
\hline
\end{tabular}
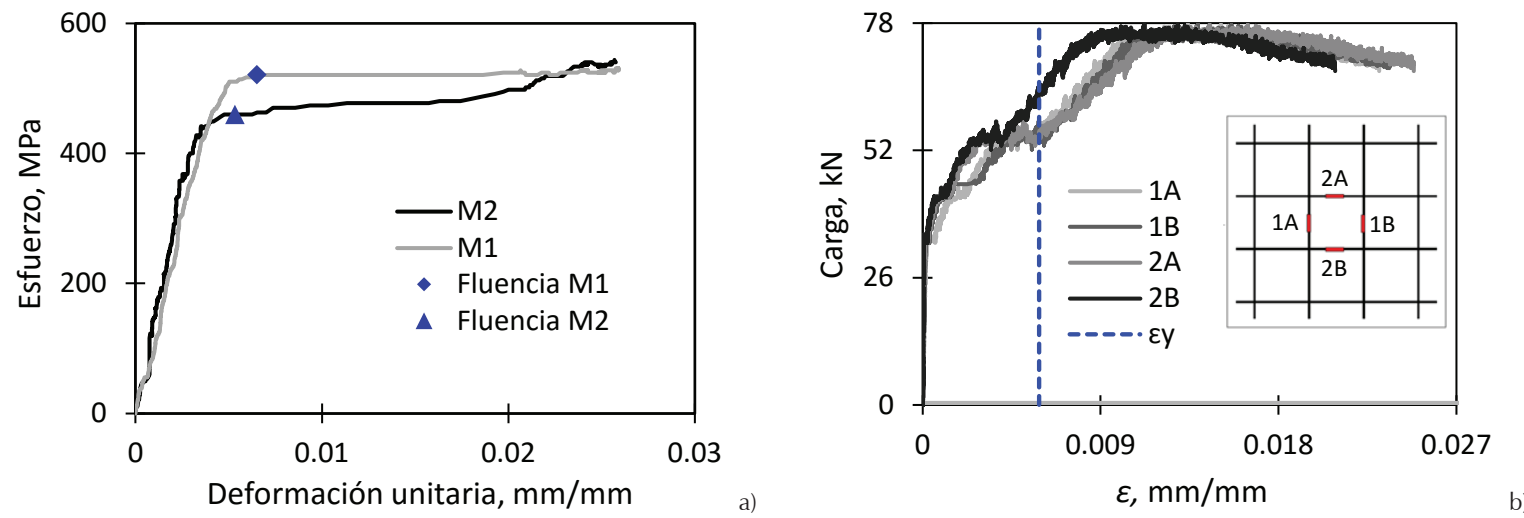

Figura 4. Curvas de comportamiento: a) curvas esfuerzo-deformación de los alambres de la malla, b) curva cargadeformación de los alambres de la malla en losa de CR

Tabla 3. Propiedades y características medidas de la malla electrosoldada

\begin{tabular}{lcc}
\hline \multicolumn{1}{c}{ Propiedades } & $X$ & $C V, \%$ \\
\hline Esfuerzo de fluencia, $f_{y^{\prime}}$ MPa & 490 & 8.8 \\
Deformación de fluencia, $\varepsilon_{y}$ & 0.0059 & 14.1 \\
Resistencia a tensión, $f_{u^{\prime}} \mathrm{MPa}$ & 545 & 3.0 \\
Deformación última, $\varepsilon_{u}$ & 0.0262 & 1.6 \\
Reducción de área, $\%$ & 40.4 & 1.9 \\
Alargamiento, $\%$ & 5.7 & 8.8 \\
Doblado $90^{\circ}, \mathrm{Si} / \mathrm{No}$ & $\mathrm{Si}$ & - \\
\hline
\end{tabular}
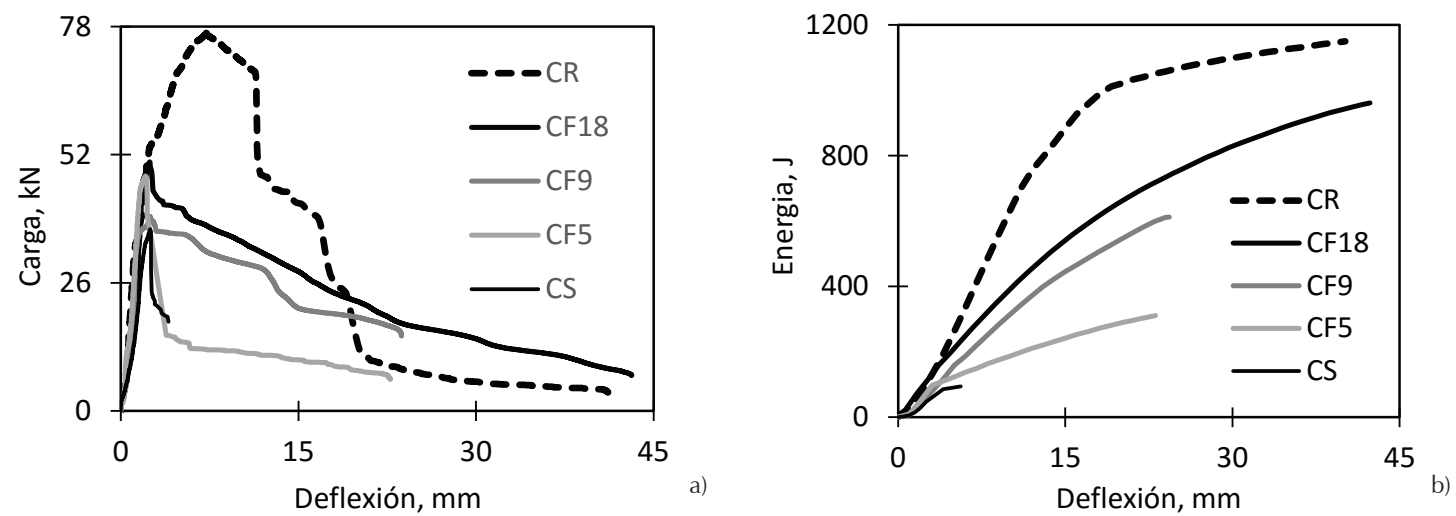

Figura 5. Curvas de desempeño de las losas: a) curvas carga-deflexión, b) curvas energía-deflexión 
DOI: https://doi.org/10.1016/j.riit.2016.11.009

Carrillo Julián, Silva Diego, Sánchez Martha

Tabla 4. Parámetros principales del ensayo a flexión de losas

\begin{tabular}{|c|c|c|c|c|c|c|c|}
\hline \multicolumn{2}{|c|}{ Nomenclatura } & CS & CF-5 & CF-9 & CF-18 & $\begin{array}{c}\text { CR } \\
\text { (Malla) }\end{array}$ & $\begin{array}{l}\text { Valor de } V_{f,} \times\left(l_{f} / d_{f}\right) \\
\text { para } T \text { igual a CR }\end{array}$ \\
\hline \multicolumn{2}{|c|}{$D_{f}, \mathrm{~kg} / \mathrm{m}^{3}$} & 0 & 3.4 & 7.6 & 14.1 & 0 & \\
\hline \multicolumn{2}{|c|}{$V_{f,} \%$} & 0 & 0.04 & 0.10 & 0.18 & 0 & \\
\hline \multicolumn{2}{|c|}{$V_{f_{s}} \times\left(l_{f} / d_{f}\right)$} & 0 & 1.92 & 4.80 & 8.64 & 0 & \\
\hline \multicolumn{2}{|c|}{$\rho_{\min }$} & 0 & 0 & 0 & 0 & $0.18 \%$ & \\
\hline \multirow{2}{*}{$P_{\max ,} \mathrm{kN}$} & $X$ & 36.5 & 46.5 & 39.8 & 46.9 & 77.7 & \\
\hline & $C V, \%$ & 1.6 & 3.7 & 1.1 & 11.0 & - & \\
\hline \multirow{2}{*}{$\delta_{\max } \mathrm{mm}$} & $X$ & 2.3 & 1.4 & 2.4 & 4.6 & 7.2 & \\
\hline & $C V, \%$ & 9.7 & 63.9 & 8.9 & 60.8 & - & \\
\hline \multirow{2}{*}{$P_{u}, \mathrm{kN}$} & $X$ & 18.9 & 5.5 & 16.1 & 7.1 & 3.7 & \\
\hline & $\mathrm{CV}, \%$ & 6.2 & 24.4 & 7.8 & 1.8 & - & \\
\hline \multirow{2}{*}{$\delta_{u^{\prime}} \mathrm{mm}$} & $X$ & 4.1 & 21.8 & 24.3 & 43.7 & 41.1 & \\
\hline & $\mathrm{CV}, \%$ & 19.1 & 6.9 & 0.6 & 1.9 & - & \\
\hline \multirow{2}{*}{$T_{5 m m^{\prime}} \mathrm{J}$} & $X$ & 100.8 & 122.2 & 144.3 & 191.5 & 258.6 & \multirow{2}{*}{15.2} \\
\hline & $\mathrm{CV}, \%$ & 13.8 & 0.6 & 12.3 & 12.9 & - & \\
\hline \multirow{2}{*}{$T_{10 m m v} \mathrm{~J}$} & $x$ & - & 186.8 & 274.8 & 380.7 & 628.2 & \multirow{2}{*}{11.1} \\
\hline & $\mathrm{CV}, \%$ & & 0.7 & 19.4 & 3.5 & - & \\
\hline \multirow{2}{*}{$T_{15 m m^{\prime}} \mathrm{J}$} & $x$ & & 240.7 & 387.2 & 535.4 & 883.9 & \multirow{2}{*}{16.3} \\
\hline & $C V, \%$ & - & 0.5 & 21.1 & 1.4 & - & \\
\hline \multirow{2}{*}{$T_{20 m m^{\prime}} \mathrm{J}$} & X & & 280.4 & 473.1 & 647.2 & 1020.3 & \multirow{2}{*}{15.1} \\
\hline & $\mathrm{CV}, \%$ & - & 4.2 & 21.2 & 2.6 & - & \\
\hline \multirow{2}{*}{$T_{25 m m^{\prime}} \mathrm{J}$} & $x$ & & & & 732.9 & 1066.3 & \\
\hline & $\mathrm{CV}, \%$ & - & - & - & 3.5 & - & \\
\hline \multirow{2}{*}{$T_{30 m m^{\prime}} \mathrm{J}$} & $X$ & & & & 802.8 & 1098.4 & \\
\hline & $\mathrm{CV}, \%$ & - & - & - & 4.5 & - & \\
\hline \multirow{2}{*}{$T_{35 m m^{\prime}} \mathrm{J}$} & $X$ & & & & 862.4 & 1125.9 & \\
\hline & $C V, \%$ & - & - & - & 4.7 & - & \\
\hline \multirow{2}{*}{$T_{40 m m} \mathrm{~J}$} & $X$ & & & & 913.8 & 1148.9 & \\
\hline & $\mathrm{CV}, \%$ & - & - & - & 4.4 & - & \\
\hline \multirow{4}{*}{$T_{l}, \mathrm{~J}$} & $X$ & 107.1 & 293.7 & 620.0 & 937.7 & 1149.5 & \\
\hline & $\mathrm{CV}, \%$ & 16.7 & 8.2 & 1.9 & 3.5 & - & \\
\hline & & & & & & $X$ & 14.4 \\
\hline & & & & & & $C V, \%$ & $13.9 \%$ \\
\hline
\end{tabular}
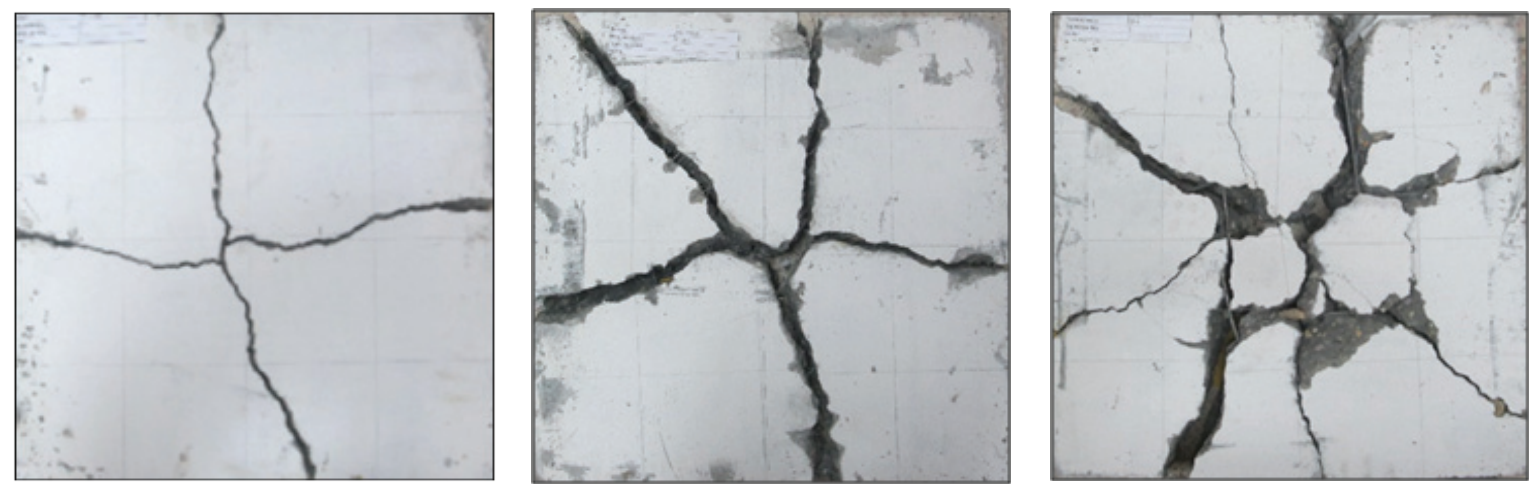

Figura 6. Esquemas de agrietamiento final de las losas: a) CS, b) $D_{f}=14.1 \mathrm{~kg} / \mathrm{m}^{3}$, c) CR 
el momento en el cual se observó una pérdida abrupta de la capacidad de resistencia.

En la figura 7 se presenta la relación entre los principales parámetros medidos en el ensayo a flexión de losas, y el producto del volumen de fibras y relación de esbeltez de las fibras $\left[V_{f} \times\left(l_{f} / d_{f}\right)\right]$. En la figura 7 y en la tabla 4 se observa que la carga máxima, $P_{\max }$ de los especímenes de CR fue $65.7 \%(77.7 \mathrm{kN} / 46.9 \mathrm{kN})$ mayor que la carga de los especímenes con $D_{f}=14.1 \mathrm{~kg} /$ $\mathrm{m}^{3}$ (DF-18). Este incremento significativo de resistencia se originó por la presencia y configuración de los alambres de acero en la malla. Sin embargo, en la figura 4 a se observa que aunque la capacidad de resistencia de la losa reforzada con malla fue mayor a la resistencia de la losa reforzada con fibras de acero y con $D_{f}=14.1 \mathrm{~kg} / \mathrm{m}^{3}$, la degradación de resistencia de la losa con fibras fue menor para deflexiones mayores a $20 \mathrm{~mm}$. En la figura 7 se observa que la deflexión en $P_{\max }\left(\delta_{\max }\right)$ y la deflexión en $P_{u}\left(\delta_{u}\right)$ aumentaron a medida que se incrementó el producto $\left[V_{f} \times\left(l_{f} / d_{f}\right)\right]$. Adicionalmente, en la figura se observa que con excepción de la deflexión $\delta_{u}$ de la losa de concreto reforzada con fibras de acero y con $D_{f}=14.1 \mathrm{~kg} / \mathrm{m}^{3}$, los principales parámetros de las losas de concreto reforzadas con malla electrosoldada, fueron mayores que los medidos en las losas reforzadas con fibras de acero.

La absorción de energía o tenacidad se representó por el área bajo la curva carga-deflexión y se determinó por medio del método de los trapecios. En la figura $5 \mathrm{~b}$ se muestra la variación de la energía en función de la deflexión en el centro del claro de las losas. En la figura se observa que a medida que aumenta la dosificación de fibras de acero, la absorción de energía aumenta, debido a que las fibras generan un mayor número de micro-fisuras y un efecto de "enganche" que permite que se generen anchos de grietas significativos antes que se presente la falla por extracción de las fibras (figura 6). En la figura $5 \mathrm{~b}$ se observa que las losas reforzadas con $14.1 \mathrm{~kg} / \mathrm{m}^{3}$ de fibras de acero mostraron valores de tenacidad más cercanos, pero menores, a las losas con refuerzo convencional. A par- tir de las curvas tenacidad-deflexión fue posible observar la variación de la absorción de energía con el producto $V_{f} \times\left(l_{f} / d_{f}\right)$ para diferentes valores de deflexión. En la tabla 4 también se indican los valores promedio de la energía para diferentes valores de deflexión en el centro del claro $(5,10,15,20,25,30,35$ y $40 \mathrm{~mm}$ ) y para el momento de la falla de la losa, $\mathrm{T}_{1}$. En la figura 8 se muestran las tendencias de energías para las deflexiones de 5, 10, 15 y $20 \mathrm{~mm}$.

En la figura $5 \mathrm{~b}$ se observa que las losas con dosificación de $14.1 \mathrm{~kg} / \mathrm{m}^{3}$ (DF-18) muestran valores de tenacidad más cercanos a los de las losas con refuerzo convencional. Sin embargo, este contenido de fibras de $14.1 \mathrm{~kg} / \mathrm{m}^{3}$ no es suficiente para igualar la tenacidad observada en la losa con malla electrosoldada en las diferentes deflexiones.

A partir de un análisis de regresión lineal se estimó el valor del producto $V_{f} \times\left(l_{f} / d_{f}\right)$ que sería necesario para igualar la energía de la losa con refuerzo convencional de malla electrosoldada, para un nivel determinado de desempeño en términos de deflexión (5, 10, 15 y $20 \mathrm{~mm})$. Como se muestra en la figura 8 , este valor de $V_{f} \times\left(l_{f} / d_{f}\right)$ corresponde al punto de cruce entre la línea de energía de la losas de CR con la línea de regresión lineal para las losas reforzadas con fibras de acero. Los valores de $V_{f,} \times\left(l_{f} / d_{f}\right)$ para las deflexiones de $5 \mathrm{~mm}, 10 \mathrm{~mm}, 15 \mathrm{~mm}$ y $20 \mathrm{~mm}$ son 15.2, 11.1, 16.3 y 15.1, respectivamente; es decir, el producto $V_{f} \times\left(l_{f} / d_{f}\right)$ varía entre 11.1 y 16.3. Tomando en cuenta que no se observa una tendencia clara de variación en función de la deflexión, se obtuvo el promedio y el $C V$ de estos valores. Como se observa en la tabla 4 , el valor promedio de $V_{f,} \times\left(l_{f} / d_{f}\right)$ para igualar la tenacidad a flexión observada en la losa reforzada con la cuantía mínima por contracción y temperatura es 14.4 y el CV es $13.9 \%$. De esta manera, para la fibra de acero utilizada en este estudio $\left(l_{f} / d_{f}=48\right)$, el valor de $V_{f}$ es de $0.3 \%$ (14.4/48), la cual corresponde a una dosificación aproximada de $24 \mathrm{~kg} / \mathrm{m}^{3}$. En general, el contenido de fibra de acero que podría remplazar la malla electrosoldada varía entre 18.0 y $26.5 \mathrm{~kg} / \mathrm{m}^{3}$ para una fibra con $l_{f} / d_{f}=48$. Si se utiliza una fibra de acero con $l_{f} / d_{f}=80$, el valor de la dosificación es aproximadamente de $14 \mathrm{~kg} / \mathrm{m}^{3}$.
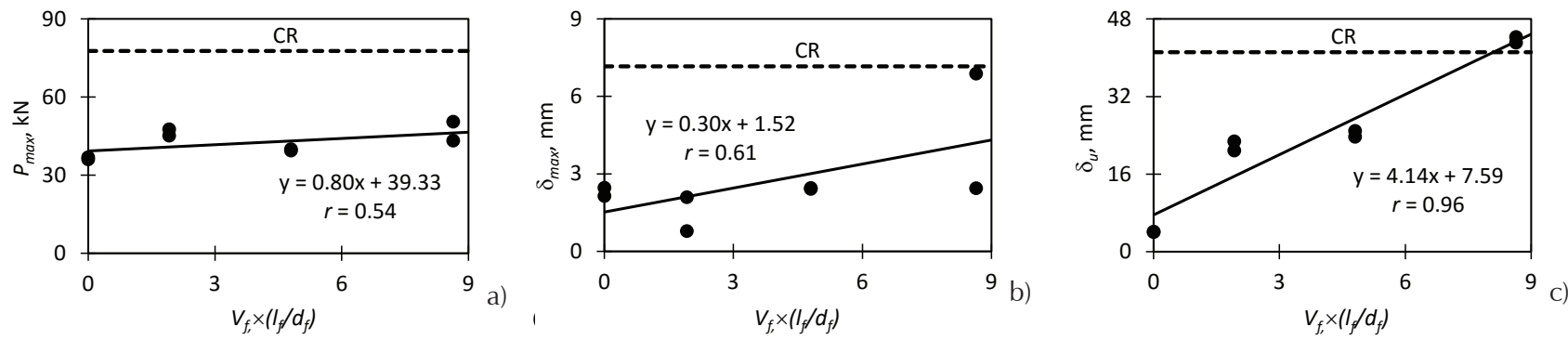

Figura 7. Tendencias de propiedades mecánicas a flexión de losas 

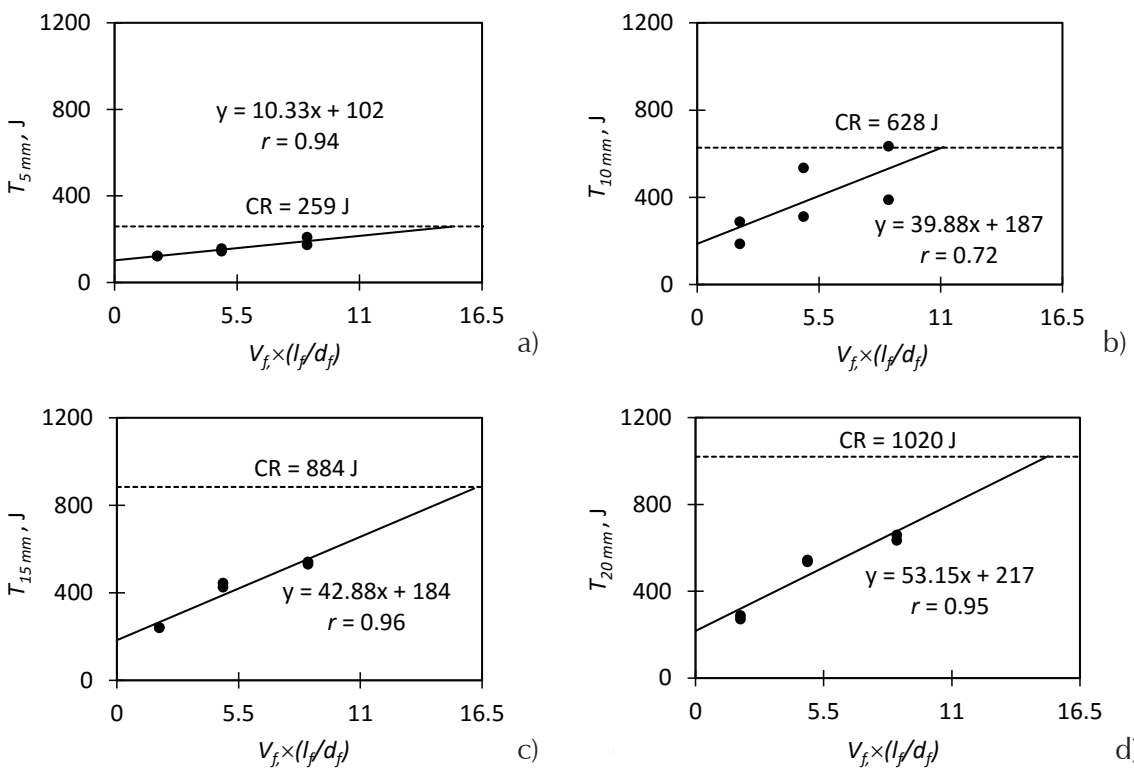

Figura 8. Absorción de energía o tenacidad para diferentes valores de deflexión: a) $5 \mathrm{~mm}$, b) $10 \mathrm{~mm}$, c) $15 \mathrm{~mm}$, d) $20 \mathrm{~mm}$

\section{Conclusiones}

En este artículo se ha evaluado experimentalmente el desempeño a flexión de losas sobre terreno para viviendas, con el fin de estimar la dosificación de fibras de acero necesaria para obtener un desempeño equivalente al de la losa de concreto reforzada con la cuantía mínima por contracción y temperatura establecida en los Reglamentos ACI 318-11 y NSR-10 (0.18\%). El programa experimental incluyó el ensayo de 10 losas cuadradas de 600 $\mathrm{mm}$ de lado y $100 \mathrm{~mm}$ de espesor construidas con concreto simple, concreto reforzado con malla electrosoldada y concreto reforzado con tres dosificaciones de fibra de acero $\left(5,9\right.$ y $\left.18 \mathrm{~kg} / \mathrm{m}^{3}\right)$. En el estudio no se evaluó el efecto del tipo de suelo sobre el comportamiento de la losa.

Las curvas tenacidad-deflexión evidenciaron que, aunque la tenacidad para la deflexión última de las losas reforzadas con malla electrosoldada y con la dosificación de fibras de $14.1 \mathrm{~kg} / \mathrm{m}^{3}$ fue similar, el comportamiento de los elementos fue significativamente diferente. En las losas reforzadas con malla electrosoldada, la tasa de degradación de resistencia fue mayor, el colapso fue más rápido y las grietas se empezaron a notar más tarde que en los especímenes reforzados con fibras de acero. Por tanto, las fibras de acero podrían generar un modo de falla de las losas de concreto más dúctil que el refuerzo con malla electrosoldada, que es un desempeño fundamental en elementos de concreto reforzado.

En el estudio se han propuesto valores de dosificación asociada a diferentes valores de deflexión. Por tanto, el diseñador puede seleccionar la dosificación de fibra que debe utilizar en función de la deflexión máxima es- perada en la losa. Estas dosificaciones se limitan al desempeño equivalente de una losa con cuantía de refuerzo de $0.18 \%$, la cual se ha proporcionado por medio de una malla electrosoldada corrugada de diámetro de $5 \mathrm{~mm}$ y aberturas cuadradas de $150 \times 150 \mathrm{~mm}$. Para estudios futuros se recomienda utilizar dosificaciones de fibras de acero entre 18.0 y $26.5 \mathrm{~kg} / \mathrm{m}^{3}$ y fibras con $l / d_{f}$ mayores a $48 \mathrm{y}$, de esta manera, robustecer la base de datos con dosificaciones y fibras de acero diferentes a las utilizadas en este estudio. Estudios futuros pueden incluir el efecto del espesor de la losa y el tipo de suelo de cimentación, así como el análisis de costo/beneficio de reemplazar la malla electrosoldada por las fibras de acero.

\section{Agradecimientos}

Los autores agradecen a la Vicerrectoría de Investigaciones de la Universidad Militar Nueva Granada (UMNG, Colombia) por la financiación del proyecto ING-1536. Adicionalmente, los autores expresan su agradecimiento a las empresas Argos y Proalco (representante de Bekaert en Colombia), en especial a los Ingenieros Diego Velandia y Liliana Cardona. De la misma forma, al ingeniero Felipe Riveros por su apoyo en la realización de las pruebas en los Laboratorios de Agregados y Concretos de la UMNG.

\section{Referencias}

ACI Committee 318 (2011). Building code requirements for structural concrete and commentary (ACI 318-11). American Concrete Institute, Farmington Hills, MI, USA. 
ACI Committee 544 (2009). Design considerations for steel fiber reinforced concrete (ACI 544.4R-88). American Concrete Institute, Farmington Hills, MI, USA.

ASTM A-82 (2001). Standard specification for steel wire, plain, for concrete reinforcement. Libro annual, USA.

Banthia N. Concrete Reinforced with deformed steel fibers, Part II: Toughness characterization. ACI Materials Journal, volumen 92 (número 2), 1995: 146-154.

Carrillo J. y Silva D. Ensayos a flexión de losas de concreto sobre terreno reforzadas con fibras de acero. Revista Ingeniería, Investigación y Tecnología, volumen 17 (número 3), 2016: 317-330.

Carrillo J., Riveros F., LLano L. Dispositivo para sujeción de múltiples transductores de desplazamiento en ensayos de losas de concreto. Ingeniería Mecánica, Tecnología y Desarrollo, volumen 5, (número 2), 2015: 313-319.

Hadi M. An investigation of the behavior of steel and polypropylene fiber reinforced concrete slabs, en: 7th International Conference: Concrete, Construction's sustainable option, Escocia, 2008.

Khalloo A. y Afshari M. Flexural behavior of small steel fiber reinforced concrete slabs. Cement and concrete Composites, volumen 27 (número 1), 2005: 141-149.

Lugo G. Fibras de acero para la construcción. Construcción y tecnología: Una isla de tecnología, Productividad y confort. Revista IMCYC, (número 216), 2007.

Nguyen M., RovnákM Q., Nguyenkim K. Punching shear resistance of steel fiber reinforced concrete flat slabs. Conference on Structural Engineering, volumen 14, 2011, pp. 1830-1837.

NMX B-253. Alambre de acero liso o corrugado para refuerzo de concreto - Especificaciones y métodos de prueba. CANACERO, México DF, 2006.
NTC 1907. Alambre de acero al carbono grafilado para refuerzo de concreto. ICONTEC, Bogotá, Colombia, 2003.

NTC 3353. Definiciones y métodos para los ensayos mecánicos de productos de acero. ICONTEC, Bogotá, Colombia, 1997.

NTC 5721. Método de ensayo para la determinación de la capacidad de absorción de energía (Tenacidad) de concreto reforzado con fibra. ICONTEC, Bogotá, Colombia, 2009.

Pigeon M. y Cantin R. Flexural properties of steel fiber-reinforced concretes at low temperatures. Cement and Concrete Composites, volumen 20 (número 5), 1998: 365-375.

NSR-10. Reglamento colombiano de Construcción Sismo Resistente. Asociación Colombiana de Ingeniería Sísmica (AIS), Bogotá, Colombia, 2010.

Sorelli L., Meda A., Plizzari G., Rossi B. Experimental investigation on slabs on grade: Steel fibers vs conventional reinforcement, en: 6th International RILEM Symposium on Fibre Reinforced Concretes, Varenna, Italia, 2004, pp. 1083-1092.

\section{Este artículo se cita: \\ Citación estilo Chicago}

Carrillo, Julian, Diego Silva, Martha Sánchez. Desempeño de losas de concreto sobre terreno reforzadas con malla electrosoldada o fibras de acero. Ingeniería Investigación y Tecnología, XVII, 04 (2016): 499-510.

\section{Citación estilo ISO 690}

Carrillo J., Silva D., Sánchez M. Desempeño de losas de concreto sobre terreno reforzadas con malla electrosoldada o fibras de acero. Ingeniería Investigación y Tecnología, volumen XVII (número 4), octubre-diciembre 2016: 499-510.

\section{Semblanzas de los autores}

Julián Carrillo. Obtuvo los títulos de ingeniero civil en 2002 por la Universidad Militar Nueva Granada, UMNG, Colombia, el de maestro en ingeniería civil (área de estructuras y sísmica) en 2004 por la Universidad de los Andes, Colombia, y el de doctor en ingeniería (área de estructuras) en 2010 por la Universidad Nacional Autónoma de México, UNAM. Actualmente es profesor asociado y director del Grupo de investigación estructuras y sísmica de la UMNG. Es miembro de tres comités del American Concrete Institute, ACI: 314, Diseño simplificado de edificios; 369, Reparación y rehabilitación sísmica; y 374, Diseño sísmico basado en desempeño de edificios de concreto.

Diego Silva. Obtuvo el título de ingeniero civil en 2015 por la Universidad Militar Nueva Granada, UMNG, Colombia. Actualmente es asistente graduado del Grupo de investigación estructuras y sísmica de la UMNG.

Martha Sánchez. Obtuvo los títulos de ingeniero civil en 1989 por el Instituto Superior Politécnico José Antonio Echeverría, Cuba, el de maestra en ingeniería civil (área de estructuras) en 2002 por la Pontificia Universidad Católica de Rio de Janeiro, Brasil, y el de 